\title{
Transcriptome profiling and comparison of maize ear heterosis during the spikelet and floret differentiation stages
}

Xiaojiao Hu${ }^{\dagger}$, Hongwu Wang ${ }^{\dagger}$, Xizhou Diao, Zhifang Liu, Kun Li, Yujin Wu, Qianjin Liang, Hui Wang and Changling Huang ${ }^{*}$

\begin{abstract}
Background: Hybridization is a prominent process in the evolution of crop plants that can give rise to gene expression variation, phenotypic novelty and heterosis. Maize is the most successful crop in utilizing heterosis. The development of hybrid maize ears exhibits strong heterotic vigor and greatly affects maize yield. However, a comprehensive perspective on transcriptional variation and its correlation with heterosis during maize ear development is not available.

Results: Using RNA sequencing technology, we investigated the transcriptome profiles of maize ears in the spikelet and floret differentiation stages of hybrid ZD808 and its parents CL11 and NG5. Our results revealed that 53.9\% $(21,258)$ of maize protein-coding genes were transcribed in at least one genotype. In both development stages, significant numbers of genes were differentially expressed between the hybrid and its parents. Gene expression inheritance analysis revealed approximately $80 \%$ of genes were expressed additively, which suggested that the complementary effect may play a foundation role in maize ear heterosis. Among non-additively expressed genes, NG5-dominant genes were predominant. Analyses of the allele-specific gene expression in hybrid identified pervasive allelic imbalance and significant preferential expression of NG5 alleles in both developmental stages. The results implied that NG5 may provide beneficial alleles that contribute greatly to heterosis. Further comparison of parental and hybrid allele-specific expression suggested that gene expression variation is largely attributable to cisregulatory variation in maize. The cis-regulatory variations tend to preserve the allelic expression levels in hybrid and result in additive expression. Comparison between the two development stages revealed that allele-specific expression and cis-/trans-regulatory variations responded differently to developmental cues, which may lead to stage-specific vigor phenotype during maize ear development.
\end{abstract}

Conclusion: Our research suggests that cis-regulated additive expression may fine-tune gene expression level into an optimal status and play a foundation role in maize ear heterosis. Our work provides a comprehensive insight into transcriptional variation and its correlation with heterosis during maize ear development. The knowledge gained from this study presents novel opportunity to improve our maize varieties.

Keywords: Maize (Zea mays L.), Ear development, Heterosis, Transcriptomics, Additive gene expression, Allelespecific expression, cis- and trans-regulation

\footnotetext{
* Correspondence: huangchangling@caas.cn

${ }^{\dagger}$ Equal contributors

National Key Facility for Crop Gene Resources and Genetic Improvement,

Institute of Crop Science, Chinese Academy of Agricultural Sciences, No.12

Zhongguancun South Main Street, Beijing 100081, China
} 


\section{Background}

Heterosis refers to the superior performance in biomass, yield, or other agronomic traits of hybrids relative to their parents $[1,2]$. This phenomenon has revolutionized crop breeding and production by increasing yields from 15 to $50 \%[3,4]$. However, the genetic and molecular bases of heterosis are controversial. Various models have been posited to explain heterosis, including classic dominance, over-dominance and epistasis models, which have been debated for over 100 years [5-9]. However, none of the hypotheses can fully explain this important scientific phenomenon. With the development of omics technologies, studies in hybrids using genomic, transcriptomic, epigenomic and proteomic approaches have provided a new perspective into the molecular mechanisms of heterosis $[1,10,11]$.

Recent researches have shown that variations in gene expression and regulatory networks are important sources of phenotypic novelty and are associated with heterosis. Comparative gene expression profiling between hybrids and their parents has been conducted in various organisms, including Arabidopsis [12, 13], maize [14-16], rice [17, 18] and other species [19]. Multiple modes of gene action, including additive, high- and lowparent dominance and over- and under-dominance were suggested to contribute to heterosis [15]. Several studies in maize have revealed that additive gene expression was prevalent and positively correlated with heterosis and high yield [14-16, 20]. However, in other studies, dominant or transgressive (over- and under-dominant) gene expression were suggested to be important in conferring superiority hybrid traits [21-24].

Hybridization gives rise to a vast reservoir of allelic variation that has been suggested to affect gene expression levels $[2,25,26]$. Early studies indicated that up to $50 \%$ of differentially expressed genes are affected by allele variations [27]. The interactions of two parent alleles in the hybrid are considered to be important determinants of a superior phenotype, and they can be regulated by cis- or trans-acting factors [28, 29]. Cis-regulation can occur as a result of variation in DNA sequences or epigenetic modifications of cis-regulatory elements of the nearby gene. Alternatively, trans-regulation is due to variation in remote trans-acting factors, which affect downstream gene expression levels. Cis-regulation changes affect gene expression in an allele-specific manner, whereas trans-regulation affects both alleles in the hybrid. The relevant contributions of cis and trans effects to the divergence of gene expression have been discussed in previous studies. In maize, cis-regulatory variation has been found to contribute greatly to parental expression divergence and is correlated with additive expression patterns in the hybrid [30, 31]. Allele-specific expression studies in maize hybrid seedlings revealed that cis-regulatory variation accounts for $70 \%$ of the differentially expressed genes [27]. In certain other species, trans-regulation has been suggested to play important roles in gene expression variation [32, 33]. However, recent evidence indicates that gene expression stability is also maintained by the coordination of cis- and trans-regulatory activity [34, 35]. These discrepancies suggests that different gene expression patterns and regulatory mechanisms may not be solely responsible for heterosis and more likely associated with particular species, tissues and developmental stages.

Maize is the most widely grown and highest-yielding crop worldwide. Immature maize ear development exhibits strong heterosis in ear architectural traits and greatly affects maize yield [36]. Ear inflorescence differentiation is a continuous, dynamic process that includes growth cone elongation, spikelet differentiation, floret differentiation and organ formation. The spikelet and floret differentiation stages are crucially important because the axillary spikelet pair meristems and floral meristems are formed during these stages, and these steps determine the two main components of maize yield, namely, kernel row number and kernel number per row $[37,38]$. Study of the molecular basis of ear heterosis during these two stages could have great impact on high-yield maize breeding.

ZD808 is an excellent maize hybrid, which was bred by our research group and approved by the National Crop Variety Approval Committee of China in 2006. This variety has been recommended by the Ministry of Agriculture as leading variety for southwestern China for eight consecutive years. ZD808 exhibits strong heterosis in ear architectural traits, with large ears, large grains and high grain yield (Table 1), and it is an excellent model for the molecular investigation of ear heterosis. ZD808 was derived from a cross between the inbred lines CL11 and NG5. The maternal line CL11 has tropical genetic components and exhibits a high resistance to stress and disease, whereas the paternal line NG5 has large ears and a high, stable yield.

In this study, using RNA sequencing technology, we presented a global gene expression profile of immature ears of maize hybrid ZD808 and its parental lines during the spikelet and floret differentiation stages. We investigated the gene expression divergence, allele-specific expression patterns and the cis- and trans-regulatory mechanisms underlying maize ear heterosis, and we compared the gene expression and regulation between the two developmental stages. Our research provides a comprehensive perspective on the transcriptomic changes and their correlations with heterosis during maize ear development. 
Table 1 Heterosis analysis of ear architectural traits of ZD808

\begin{tabular}{|c|c|c|c|c|c|c|}
\hline Stage & Trait & $N G 5^{a}$ & $\mathrm{CL} 11^{\mathrm{a}}$ & ZD $808^{\mathrm{a}}$ & MPH (100\%) & $\mathrm{BPH}(100 \%)$ \\
\hline \multirow[t]{2}{*}{ Spikelet differentiation stage } & ear length $(\mathrm{mm})$ & $8.25 \pm 1.07$ & $6.4 \pm 0.36$ & $15.58 \pm 0.69$ & $112.7^{* *}$ & $88.85^{* *}$ \\
\hline & ear diameter (mm) & $2.02 \pm 0.19$ & $1.42 \pm 0.06$ & $2.86 \pm 0.32$ & $66.28^{* *}$ & $41.58^{* *}$ \\
\hline \multirow[t]{2}{*}{ Floret differentiation stage } & ear length (mm) & $14.23 \pm 0.55$ & $10.3 \pm 1.15$ & $20.67 \pm 1.53$ & $68.53^{* *}$ & $45.26^{* *}$ \\
\hline & ear diameter (mm) & $2.4 \pm 0.05$ & $1.87 \pm 0.03$ & $3.4 \pm 0.17$ & $59.25^{* *}$ & $41.67^{* *}$ \\
\hline \multirow[t]{5}{*}{ Mature ear } & ear length (cm) & $20.28 \pm 1.00$ & $15.05 \pm 0.26$ & $26.05 \pm 0.58$ & $47.49^{* *}$ & $28.48^{* *}$ \\
\hline & ear diameter (cm) & $4.68 \pm 0.20$ & $4.08 \pm 0.17$ & $5.60 \pm 0.34$ & $28^{* *}$ & $19.78^{* *}$ \\
\hline & ear row number & $15.50 \pm 1.00$ & $12.50 \pm 1.00$ & $16 \pm 0.00$ & $14.29^{* *}$ & 3.23 \\
\hline & kernel number per row & $32.25 \pm 1.71$ & $21 \pm 1.15$ & $50.75 \pm 4.03$ & $90.61^{* *}$ & $57.36^{* *}$ \\
\hline & grain yield (kg/mu) & $301 \pm 12.5$ & $230 \pm 4.5$ & $553 \pm 26.1$ & $108^{* *}$ & $84^{* *}$ \\
\hline
\end{tabular}

** indicate significant differences at $P<0.01$

${ }^{\text {a }}$ Values are means \pm standard deviation. $\mathrm{MPH}$, midparent heterosis; $\mathrm{BPH}$, Best parent heterosis

\section{a}

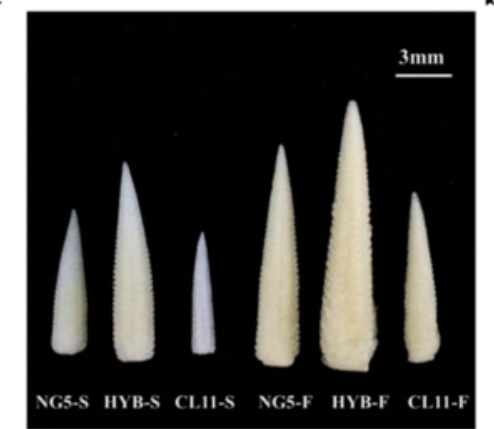

NG5
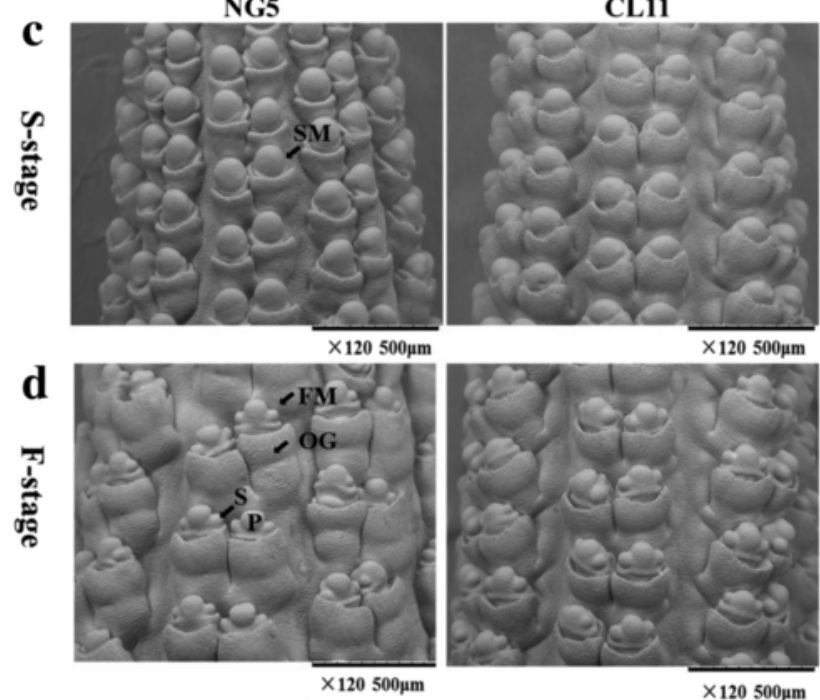

b

CL11

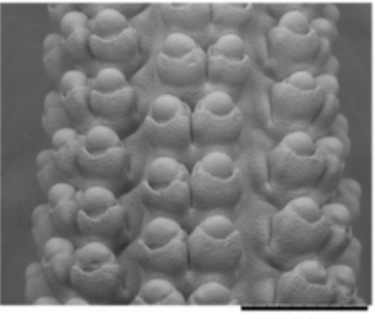

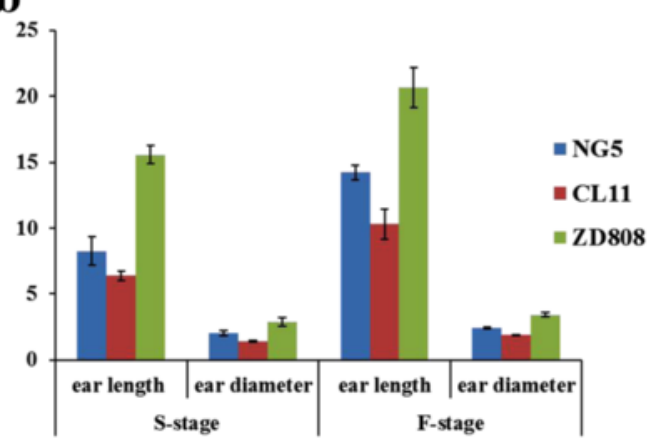

HYB
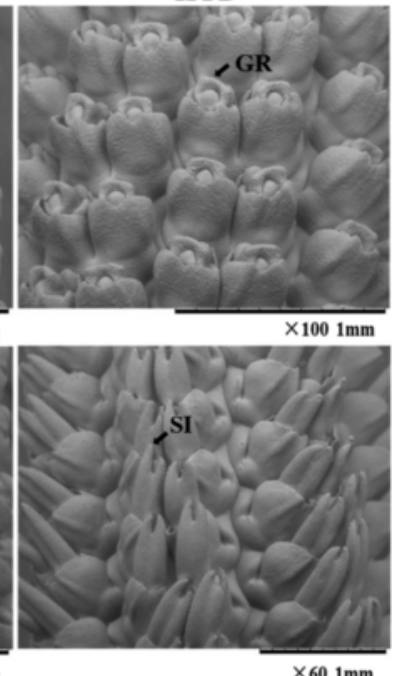

Fig. 1 Characterization of the ear architectural traits of maize hybrid ZD808 (HYB) and its parental lines. a Phenotypic differences of the immature ears of HYB, CL11 and NG5 in the spikelet and floret differentiation stages. b Ear length and diameter of HYB, CL11 and NG5 in the spikelet and floret differentiation stages. Scanning electron microscope observation of immature ears of NG5, CL11 and HYB in spikelet (c) and floret differentiation stages (d). Abbreviations: S-stage, spikelet differentiation stage; F-stage, floret differentiation stage; SM, spikelet meristem; GR, Gynoecial ridge; FM, floret meristem; OG, outer glume; S, stamen primordia; P, pistil primordial; SI, silk 


\section{Results}

\section{Characterizing the ear traits of ZD808 and its parental} lines

To dissect the relationship between global gene expression changes and heterosis during ear development, the immature ears of ZD808 (HYB) and its parent lines in spikelet and floret differentiation stages were collected for transcriptome analysis (Fig. 1a). In the spikelet differentiation stage (S-stage), the spikelet pair primordia (SPM) arise on the flanks of the inflorescence meristem (IM) and give rise to a pair of spikelet meristems (SM) (Fig. 1c). In the floret differentiation stage (F-stage), the SMs produce two floret meristems (FM) with obvious stamen and pistil primordia, (Fig. 1d). These two phases are crucial for ear development and heterosis formation. Further observation revealed that ear development in hybrids was more vigorous than in the parental lines, and the paternal line NG5 showed larger ear size than the maternal line CL11 (Fig. 1a and b). Significant midparent heterosis $(\mathrm{MPH})$ and best-parent heterosis $(\mathrm{BPH})$ $(p<0.01)$ were discovered for ear length and ear diameter in the S- and F-stages, and the $\mathrm{MPH}$ and $\mathrm{BPH}$ values were higher in the S-stage (Table 1). Furthermore, we also observed significant $\mathrm{MPH}$ and $\mathrm{BPH}(p<0.01)$ for the ear length, ear diameter, kernel number per row and grain yield at the mature stage (Table 1). These results indicate that ZD808 displays a strong hybrid vigor than both parents and the degree of heterosis for ear traits was larger in the spikelet differentiation stage.

\section{Deep sequencing and mapping of maize inbred and hybrid transcriptomes}

cDNA libraries of immature ears of ZD808 and its parental inbred lines CL11 and NG5 in the spikelet and floret differentiation stages were prepared and sequenced on an Illumina HiSeq $2 \mathrm{G}$ platform. To increase the statistical power, two biological replicates of each sample were sequenced. After reads with low sequencing quality were filtered out, between 43.9 and 63.5 million 100-bp paired-end reads were obtained for each of the replicates and genotypes. Among these, about 80.7\% (516 million) of the total paired reads were aligned to the B73 reference genome (ZmB73_RefGen_v3) and $77.9 \%$ were mapped to unique positions. As expected, more than $97 \%$ of reads mapped to protein-coding genes, and the others were distributed among introns (0.4-1.3\%) and intergenic regions (1.2-1.7\%) (Additional file 1). Uniquely mapped reads were used to estimate transcript levels. Expression values were expressed in units of RPKM (reads per kilobase per million reads mapped). Two biological replicates were highly correlated, with an average Pearson's correlation coefficient of 0.99 (Additional file 2).
For all analyses performed in the study, only the protein-coding genes were included, which are a subset of 39,469 gene models obtained after excluding transposons, pseudogenes, contaminants, and other lowconfidence annotations. A transcript is considered to be positively expressed only if its RPKM $\geq 1$. Based on this criterion, 21,258 genes were transcribed in at least one sample, which accounted for $53.9 \%$ of maize proteincoding genes (Additional file 3). On average, 2760 and 4314 genes (13.3 and $20.8 \%$ of expressed genes) exhibited high $(\mathrm{RPKM} \geq 50)$ and medium $(20 \leq \mathrm{RPKM}<50)$ expression levels, respectively, and low expression genes accounted for $66.0 \%$ of the expressed genes (RPKM $\leq$ 20). More genes were expressed in hybrids compared with CL11 and NG5 in both developmental stages (Additional file 3).

\section{Expression divergence between hybrid and inbred parents}

To fully elucidate the gene expression divergence and its effect on heterosis, we performed pairwise comparisons between the hybrid and the parents. Differentially expressed genes (DEGs) were identified if the RPKM value of a gene was greater than or equal to 1 in at least one of the genotypes and if the adjusted $p$-value for FDR was less than 0.05 . Using this significance threshold, in the spikelet differentiation stage, we identified 12,550 genes differentially expressed between the parental lines CL11 and NG5, which accounted for $54.48 \%$ of analyzed genes $(23,038)$ (Table 2$)$. The high expression divergence confirmed the large genetic distance between the parental lines. The number of upregulated gene numbers is similar to the number of downregulated genes $(27.26 \%$ up vs. $27.22 \%$ down), which implies equal contributions of CL11 and NG5 to gene expression divergence (Table 2). Advanced comparisons between hybrids and parental lines revealed fewer DEGs than the comparison of the two parental lines. Between HYB and CL11, 8290 (35.98\%) DEGs were identified, including 4310 (18.71\%) upregulated and 3980 (17.28\%) downregulated. Between HYB and NG5, only 4309 (18.70\%) DEGs were discovered, including $10.18 \%$ upregulated and $8.52 \%$ downregulated (Table 2). These results show that the expression profile of the hybrid is more similar to the paternal line NG5 and more divergent from CL11. In addition, more genes were actively expressed in the hybrid. In the floret differentiation stage, similar differential expression statuses were also discovered; however, the DEGs from each comparison were decreased. There were 10,120 (43.93\%) differences between CL11 and NG5, 7315 (31.75\%) between HYB and CL11, and 2696 (11.70\%) between HYB and NG5 (Table 2). Gene differential expression underlies the phenotype variation; decreasing DEGs in the F-stage appropriately explained the lower $\mathrm{MPH}$ 
Table 2 Differentially expressed genes between hybrid and the parents in the spikelet and floret differentiation stages

\begin{tabular}{|c|c|c|c|c|c|c|}
\hline \multirow{2}{*}{$\begin{array}{l}\text { Comparison } \\
\text { Group }\end{array}$} & \multicolumn{2}{|l|}{ UP } & \multicolumn{2}{|l|}{ Down } & \multicolumn{2}{|l|}{ Total } \\
\hline & Gene number & Percentage & Gene number & Percentage & Gene number & Percentage \\
\hline CL11-S vs NG5-S & 6280 & $27.26 \%$ & 6270 & $27.22 \%$ & 12550 & $54.48 \%$ \\
\hline HYB-S vs CL11-S & 4310 & $18.71 \%$ & 3980 & $17.28 \%$ & 8290 & $35.98 \%$ \\
\hline HYB-S vs NG5- S & 2346 & $10.18 \%$ & 1963 & $8.52 \%$ & 4309 & $18.70 \%$ \\
\hline CL11-F vs NG5-F & 4985 & $21.64 \%$ & 5135 & $22.29 \%$ & 10120 & $43.93 \%$ \\
\hline HYB-F vs CL11-F & 4048 & $17.57 \%$ & 3267 & $14.18 \%$ & 7315 & $31.75 \%$ \\
\hline HYB-F vs NG5-F & 1475 & $6.40 \%$ & 1221 & $5.30 \%$ & 2696 & $11.70 \%$ \\
\hline CLI1-S vs CL11-F & 608 & $2.64 \%$ & 366 & $1.59 \%$ & 974 & $4.23 \%$ \\
\hline NG5-S vs NG5-F & 152 & $0.66 \%$ & 288 & $1.25 \%$ & 440 & $1.91 \%$ \\
\hline HYB-S vs HYB-F & 133 & $0.58 \%$ & 352 & $1.53 \%$ & 485 & $2.11 \%$ \\
\hline
\end{tabular}

Total analyzed genes: 23038 ; Deseq $P$ value with padj < 0.05; $\mathrm{S}$ denotes the spikelet differentiation stage; $\mathrm{F}$ denotes the floret differentiation stage

and $\mathrm{BPH}$ values of relative ear traits in the F-stage compared to the S-stage (Table 1).

Further comparison of the two development stages revealed 974, 485, and 440 DEGs in CL11-S vs. CL11-F, HYB-S vs. HYB-F, NG5-S vs. NG5-F, respectively (Table 2). Venn diagram showed that 249 genes were differentially expressed between two development stages in at least two genotypes (Additional file 2: Figure S2A). Hierarchical clustering and gene ontology (GO) enrichment revealed that these genes exhibited similar expression pattern among genotypes and mainly enriched ([FDR] $<0.01$, Yekutieli FDR dependency) in 'biological regulation' and 'developmental process' (Additional file 2: Figure S2B and S3). Under these two categories, 52 (26.1\%) and 28 (14.1\%) DEGs, respectively, were identified to be involved in regulation of ear development, including some well-known genes (Additional file 4). RAMOSA1 (RA1), RAMOSA2 (RA2), RAMOSA3 (RA3) and BRANCHED SILKLESS1 (BD1) control the determinacy and identity of the spikelet-pair meristem in maize, and they were upregulated in the spikelet differentiation stage in at least two genotypes [39-42]. BARREN INFLORESCENCE2 (BIF2) together with TEOSINTE BRANCHED 1 (TB1) affecting the initiation and maintenance of axillary meristems were also found upregulated in S-stage [43, 44]. Furthermore, ZEA FLORICAULA/LEAFY1 (ZFL1), ZFL2 and DELAYED FLOWERING1 (DLF1) genes which are required for floral transition also exhibited increased transcript levels in the S-stage $[45,46]$. While in the floret differentiation stage (F-stage), MADS box (ZMM6, ZMM7, ZMM17, ZMM18 and ZMM29), GATA and C2C2-YABBY transcription factors which are crucial for floral meristem determinacy and organ development were significantly upregulated [47-49]. EREBP-transcription factors, Auxin efflux carrier component, Aux/IAA and Auxin response factor proteins which play key roles in gibberellin and auxin response during floral meristems initiation also increased expression abundance in F-stage (Additional file 4). These results confirm the validity of our chosen development stages.

Using a Venn diagram to compare DEGs between hybrid and its parents reveals that 59.3\% (5298 and 2147) and 61.8\% (4733 and 1525) DEGs between CL11 and NG5, respectively, are differentially expressed between HYB and CL11 in the spikelet and floret differentiation stages, whereas only $30.5 \%$ (1684 and 2147 ) and 9.6\% (971 and 1525) are differentially expressed between HYB and NG5. A total of $24.7 \%$ (2147 of 12,550) and $15.1 \%$ $(1525$ of 10,120$)$ of DEGs were shared by the three comparisons (Fig. 2a and b). The results show that DEGs are common existence and differ among genotypes and developmental stages. Hierarchical clustering of DEGs showed that different developmental stages of the same genotype tend to cluster together, and the gene expression patterns in the hybrid were more similar to those in the paternal line NG5 in both developmental stages (Fig. 2c). This result corresponds to the more robust ear phenotype of NG5 observed at corresponding stages (Fig. 1a and b).

\section{Differential gene expression patterns and heterosis}

To gain overall insight into differential gene expression patterns between the F1 hybrid and its parental lines, the DEGs from two developmental stages were further classified into additive and non-additive patterns based on the pairwise comparisons between expression levels of the hybrid and the mid-parent expression value (MPV). We identified 10,093 (73.8\%) and 9014 (79.9\%) genes expressed additively in the spikelet and floret differentiation stages, respectively, whereas only 3577 (26.2\%) and $2272(20.1 \%)$ displayed a non-additive expression pattern (Table 3). The prevalence of additively expressed genes implied complementary effects on gene expression in hybrid. According to the pairwise comparison results among CL11, NG5 and HYB, the non- 

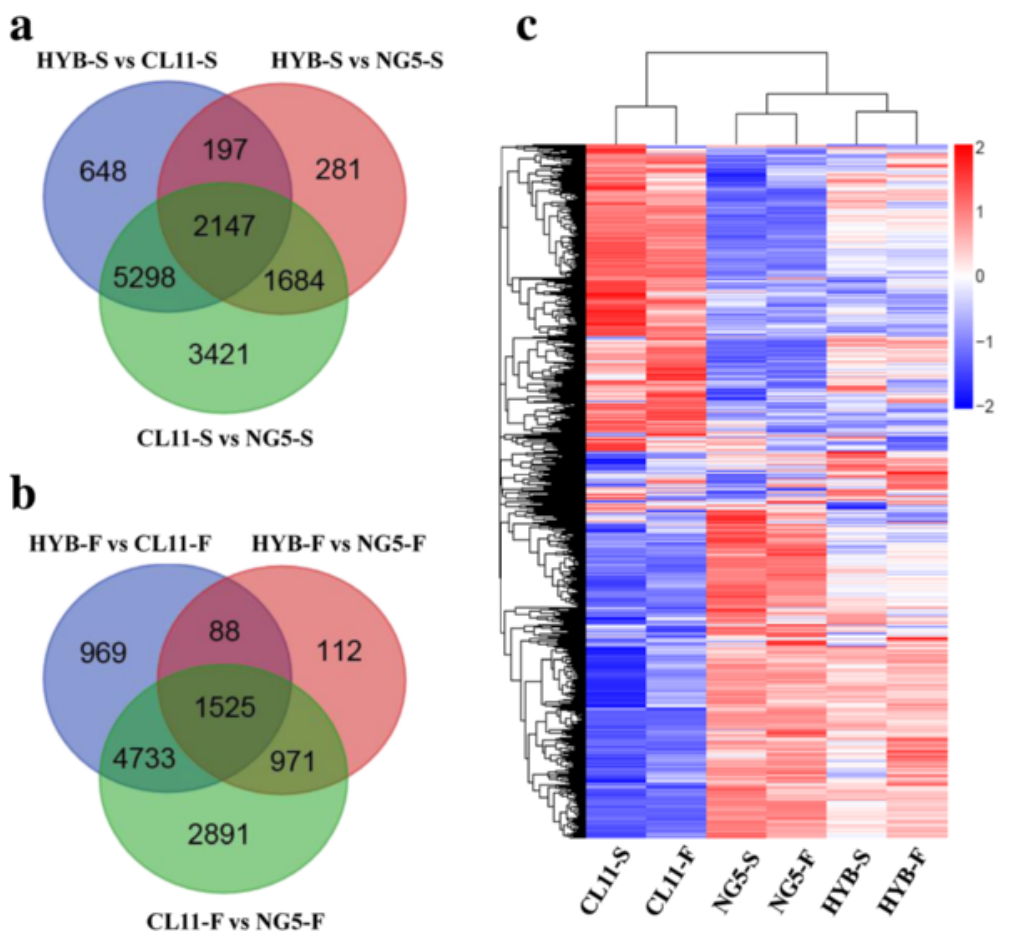

Fig. 2 Venn diagram comparison and hierarchical cluster analysis of differentially expressed genes among genotypes. Venn diagram comparison of differential expressed genes between the hybrid and its parents in the spikelet (a) and floret differentiation stages (b) of maize immature ear. $\mathbf{c}$ Hierarchical cluster analysis of differentially expressed genes among genotypes. The color key represents $\log _{10}(\mathrm{RPKM}+1)$. Red indicates high relative expression and blue indicates low relative expression. $\mathrm{S}$ denotes the spikelet differentiation stage, $\mathrm{F}$ denotes the floret differentiation stage

additively expressed genes can be further divided into (I) over-dominance, (II) high-parent dominance, (III) lowparent dominance, (IV) under-dominance and (V) conserved expression classes. The detailed gene proportions of each class of two development stages are shown in Table 3. In the spikelet differentiation stage, the parent- dominant expression classes II and III accounted for the majority $(2938,82.14 \%)$ of non-additively expressed genes; 1981 genes (55.38\%) exhibited NG5-dominant expression, and 957 genes (26.75\%) exhibited CL11-dominant expression. Only 143 (4.0\%) and 119 (3.3\%) genes showed overdominance (class I) and under-dominance (class IV),

Table 3 Classification of additive and non-additive expression patterns in hybrid

\begin{tabular}{|c|c|c|c|}
\hline \multicolumn{2}{|l|}{ Expression classes } & \multirow{2}{*}{$\frac{\text { S-Stage }}{10093(73.8 \%)}$} & \multirow{2}{*}{$\frac{\text { F-Stage }}{9014(79.9 \%)}$} \\
\hline $\operatorname{Additive~}^{a}\left(M P V=F_{1}\right)$ & & & \\
\hline Non-additive $\left(\mathrm{MPV} \neq \mathrm{F}_{1}\right)$ & & 3577 (26.2\%) & $2272(20.1 \%)$ \\
\hline । & Over-dominance $^{c}$ & 143 & 109 \\
\hline \multirow[t]{2}{*}{ II High-parent dominance } & CL11-dominance $^{\mathrm{b}}$ & 492 & 227 \\
\hline & NG5-dominance ${ }^{b}$ & 995 & 865 \\
\hline \multirow[t]{2}{*}{ III Low-parent dominance } & CL11-dominance ${ }^{b}$ & 465 & 141 \\
\hline & NG5-dominance ${ }^{b}$ & 986 & 737 \\
\hline IV & Under-dominance $^{d}$ & 119 & 27 \\
\hline V & Conserved $^{e}$ & 377 & 166 \\
\hline Total & & 13670 & 11286 \\
\hline
\end{tabular}

${ }^{a}$ based on fisher exact test between midparent value (MPV) and hybrid (qvalue < 0.05 )

${ }^{b}$ based on fisher exact test (qvalue $<0.05$ ); hybrid must be significantly different than midparent value and not significantly different from either high or low parent

cabove high parent; based on fisher exact test between high parent and hybrid (qvalue $<0.05$ )

dbelow low parent; based on fisher exact test between low parent and hybrid (qvalue $<0.05$ )

${ }^{e}$ based on fisher exact test (qvalue $<0.05$ ); hybrid value must be significantly different than midparent value and within the parental range 
respectively (Table 3 and Additional file 5). In the floret differentiation stage, 1970 genes exhibited parentdominant expression (II and III, $86.71 \%$ of 2272 nonadditive expressed genes), with 1602 (70.51\%) having NG5-dominant expression and 368 (16.20\%) showing CL11-dominant expression. A total of 109 and 27 genes displayed over-dominance and under-dominance expression patterns, respectively (Table 3 and Additional file 6). These results revealed that the majority of DEGs displayed an additive expression pattern, suggesting that a complementary effect have a fundamental role in the early formation of maize ear heterosis. Among non-additively expressed genes, a significant number of genes showed an NG5-dominant expression pattern, implying that the NG5 allele may greatly affect the gene expression levels in hybrid and also contribute to hybrid vigor.

Gene expression profiles fluctuated with developmental stage, and comparisons of gene expression patterns between the two development stages revealed that 6452 (63.9\%) additive genes from the spikelet differentiation stage maintained their additive expression status in the floret differentiation stage, whereas 952 genes changed to exhibit a non-additive expression pattern. In contrast, 998 (27.9\%) of non-additive genes maintained the expression status in the floret differentiation stage, and
1425 (39.8\%) genes changed to exhibit an additive expression pattern (Fig. 3a), which indicates that nonadditively expressed genes were more affected by the development stage.

\section{Gene Ontology (GO) analysis of additive and non-additive genes}

To ascertain the molecular and biological functions of genes with additive and non-additive expression patterns and to determine their biological roles in ear heterosis, we conducted GO enrichment analysis using single enrichments analysis from AgriGO website. In total, 6003 of 10,093 and 5350 of 9014 additively expressed genes in the spikelet and floret stages, respectively, were found to be enriched ([FDR] $<0.01$, Yekutieli FDR dependency) in GO terms from biological process (BP), molecular functions (MF) and cellular component (CC). In the BP category, the most overrepresented subcategories were 'metabolic process' (54.0 and 55.4\%), 'cellular process' (53.2 and 53.0\%) and 'biological regulation' (18.3 and 19.1\%) in both developmental stages. Further examination of specific subcategories in 'metabolic process' and 'cellular process' revealed that 'primary metabolic process' (44.6 and 44.9\%), 'cellular metabolic process' (41.4 and 41.9\%), 'macromolecule metabolic process'

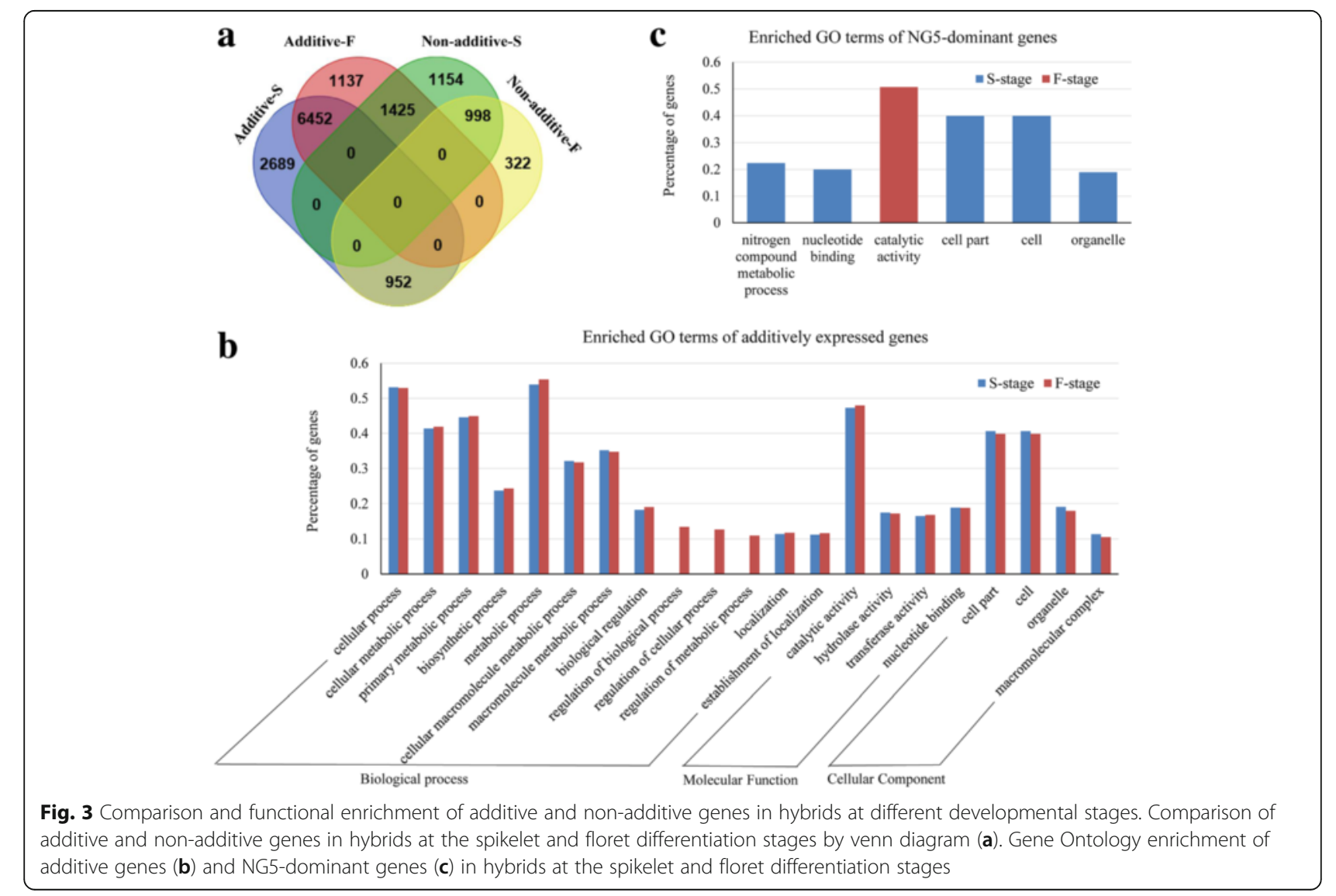


(35.2 and 34.7\%) and 'biosynthetic process' (23.7 and 24.3\%) were enriched in both sets of transcripts from the two stages. In the MF category, 'catalytic activity' (47.3 and $48.0 \%)$ and 'nucleotide binding' (18.9 and 18.9\%) were prominently represented, while 'cell' (40.6 and 39.9\%) and 'organelle' (19.2 and 18.0\%) dominated the cellular component category (Fig. 3b and Additional file 7). These results revealed that genes from the additive expression class participated in extensive biosynthetic and metabolic activities, which are required for maize ear development and early heterosis formation in both development stages. Some classical genes such as ZAG3 (GRMZM2G160565), SID1 (GRMZM2G176175), ZMM8 (GRMZM2G102161), and ZMM14 (GRMZM2G099522), which affect the fundamental formation of maize ear architecture, were also expressed additively in both stages (Additional files 5 and 6).

In contrast, non-additively expressed genes were enriched in different GO functional categories in the spikelet and floret differentiation stages. In the spikelet differentiation stage, the CL11-dominant genes were overrepresented in BP terms such as 'metabolic process' (65.6\%) and 'cellular process' (60.0\%). The NG5dominant genes were significantly enriched in the BP term 'nitrogen compound metabolic process' (22.4\%) and the MF term 'nucleotide binding' (20.0\%) (Fig. 3c and Additional file 7). Genes from the over-dominance expression class were found to be enriched in the MF term 'hydrolase activity' (29.7\%), and no GO term was significantly enriched in genes from the underdominance expression class. In the floret differentiation stage, only the NG5-dominant genes were significantly enriched in the MF term 'catalytic activity' (50.7\%) (Fig. 3c and Additional file 7). More functional GO terms were enriched in the first stage, suggesting that non-additive genes played an important role during the period when spikelet pair meristems (SPMs) give rise to spikelet meristems (SMs).

We further inspected the NG5-dominant genes due to their high representation in the non-additive expression class. In the spikelet differentiation stage, many transcription factors, including MADS-box (e.g., ZGA5, SI1), bHLH DNA-binding superfamily protein, Auxin response factor and Ethylene-responsive transcription factor were found in the "cellular process" GO term, which may trigger cell identity, regulate hormone signaling and promote the transition from SPMs to SMs. In the "nitrogen compound metabolic process" term, 35 genes were discovered that participate in glutamine synthesis (e.g., GLN4), glutamine metabolism and aspartate metabolism, which are essential for nitride assimilation and affect maize ear development and spikelet formation (Additional file 8). In the floret differentiation stage, 36 genes participated in oxidation-reduction reactions (e.g.,
GA20OX1, APX2, CAT1, and CAT3) and were found in the "catalytic activity" term, suggesting a role in stress responses and signal transduction in the floret differentiation stage (Additional file 8).

\section{Global allele-specific expression (ASE) analysis}

The transcriptional activities of different alleles in a hybrid can differ considerably, and this is an important source of the variation in gene expression. To infer hybrid ASE levels, parent-specific SNPs were detected in each parent and used to discern alleles in the hybrid. After applying quality control criteria, we found that 44,675 and 38,957 of SNPs located in gene bodies had a minimum read coverage of 10 in the hybrid at the spikelet and floret differentiation stages, respectively. A total of 12,637 and 11,993 genes, which represented 32.0 and $30.4 \%$ of protein coding genes, respectively, were marked by the filtered SNPs (Table 4). Normalized mapped readdepth coverage at SNP sites in the hybrid and parental alignments was used to quantify the expression of alleles. Allelic bias in the hybrid was identified for each SNP if the allelic ratio differed significantly from the expected allelic ratio of 1.0 (binomial exact test, adjust $p$-value $<$ 0.05 ). For convenience, we used $\mathrm{CL} 11_{\mathrm{HYB}}$ and NG5 $5_{\mathrm{HYB}}$ to represent the expression levels of the corresponding allele in the hybrid. In the spikelet differentiation stage of maize ear, 7126 genes (56.4\% of 12,637 analyzed genes) were identified as having significant allelic bias. Of these, $2514(35.3 \%)$ genes displayed $\mathrm{CL} 11_{\mathrm{HYB}}$ bias and $4612(64.7 \%)$ genes displayed NG5 $5_{\mathrm{HYB}}$ bias (Fig. 4 and Additional file 9). In the floret differentiation stage, we identified 6625 (52.4\%) ASE genes; 2967 (44.8\%) genes exhibited $\mathrm{CL} 11_{\mathrm{HYB}}$ bias and 3658 (55.2\%) genes exhibited NG5 $5_{\mathrm{HYB}}$ bias (Fig. 4 and Additional file 10). These results indicated a strong expression bias toward $\mathrm{NG5}_{\mathrm{HYB}}$ in both developmental stages, suggesting that the NG5 genome contributes greatly to gene expression in the hybrid.

According to the extent of bias, the ASE genes can be classified into three different classes: monoallelic expression (only one allele is expressed in the hybrid), preferential expression (expression level differs by more than two-fold between $\mathrm{CL} 1_{\mathrm{HYB}}$ and NG5 ${ }_{\mathrm{HYB}}$ ) and biallelic expression (expression level varies by less than two-fold between $\mathrm{CL} 11_{\mathrm{HYB}}$ and $\left.\mathrm{NG} 5_{\mathrm{HYB}}\right)$. Using these criteria, we found that 36.7 and $33.6 \%$ of ASE genes had biallelic expression, 48.6 and $47.5 \%$ showed preferential allelic expression, and 14.7 and $18.9 \%$ had monoallelic expression in the spikelet and floret development stages, respectively, which further confirmed the substantial expression preferences of parental alleles in the hybrid (Fig. 4 and Additional files 9 and 10).

Comparison of ASE in the two developmental stages revealed that among the 10,388 genes analyzed in both 
Table 4 SNPs for assessing allele-specific gene expression in hybrid in the spikelet and floret differentiation stages

\begin{tabular}{lllclcr}
\hline & SNPs for ASE analysis & Reads covered SNPs & Reads/SNP & Gene numbers & SNPs/Gene & \% of protein coding gene \\
\hline S-Stage & 44675 & 3113870 & 69.7 & 12637 & 3.5 & 32.0 \\
F-Stage & 38957 & 2647720 & 68.0 & 11993 & 3.2 & 30.4
\end{tabular}

stages, 7261 (69.9\%) genes exhibited conserved allele bias patterns in the two stages, while 495 CL11 $1_{\mathrm{HYB}}$ biased genes and $1244 \mathrm{NG5}_{\mathrm{HYB}}$ biased genes changed their expression bias. There were also 1388 genes with non-biased expression that were converted into biased allelic expression patterns (Additional file 11). These results indicate that ASE varies between developmental stages, and suggest the presence of cis-regulatory elements interacting with development cues.

\section{Cis and trans effects on gene expression divergence}

In the hybrid, gene expression divergence between parental alleles can result from changes in cis- and/or trans-regulation [30, 50]. Therefore, we compared ASE in the parental lines and the hybrid in the spikelet and floret differentiation stages to identify cis-and transregulation divergence. The expression difference between the two parental inbred lines reflected both cis and trans effects. In the hybrid, because both alleles were under the same genetic background and shared a common set of trans-regulatory factors, the allelic expression divergence in the hybrid was considered to represent cis effects. Trans effects could be detected by subtracting cis effects from the overall set of cis and trans effects. According to this classification criterion, in the spikelet differentiation stage, we identified 4869 (38.6\%) and 1107 (8.8\%) genes that showed cis only and trans only effects, respectively, whereas 1868 (14.8\%) genes were associated with both cis and trans effects (cis-trans). Over two thousand $(2476,19.6 \%)$ genes showed no significant evidence of either parental expression divergence or significant cis-or trans-regulation divergence and were classified as "conserved." A total of 2317 (18.3\%) genes had an "ambiguous" expression pattern with no clear biological interpretation (Fig. 5a and Additional file 12). The results showed that there were significantly more cis-regulatory effects than trans-regulatory effects. The 1868 genes with both cis and trans effects were further subdivided into two categories based on the acting direction of the cis and trans effects: "enhancing," in which both the cis-and trans-regulation effect favored expression of the same allele, and "compensating," in which both the cis- and trans-regulation effect favored expression of the opposite allele. Among the 1868 genes, 652 genes (34.9\%) were subjected to enhancing cis and trans interactions and 1216 genes (65.1\%) were related to compensating cis and trans interactions (Fig. 5a and Additional files 12 and 13). In the floret differentiation stage, among the 11,993 analyzed genes, 4888 (40.8\%), 549 (4.6\%), 235 (19.7\%) and $960(80.3 \%)$ genes fell into the cis only, trans only, "enhancing" and "compensating" cis-trans interactions, respectively, and 3156 genes were classified as "conserved." The remaining 2205 genes showed an "ambiguous" expression pattern and were excluded from further analysis (Fig. 5b and Additional files 12 and 14). Detailed analysis showed that a large number of cis and trans genes exhibited negative $\log _{2}$ ratios of allelic expression
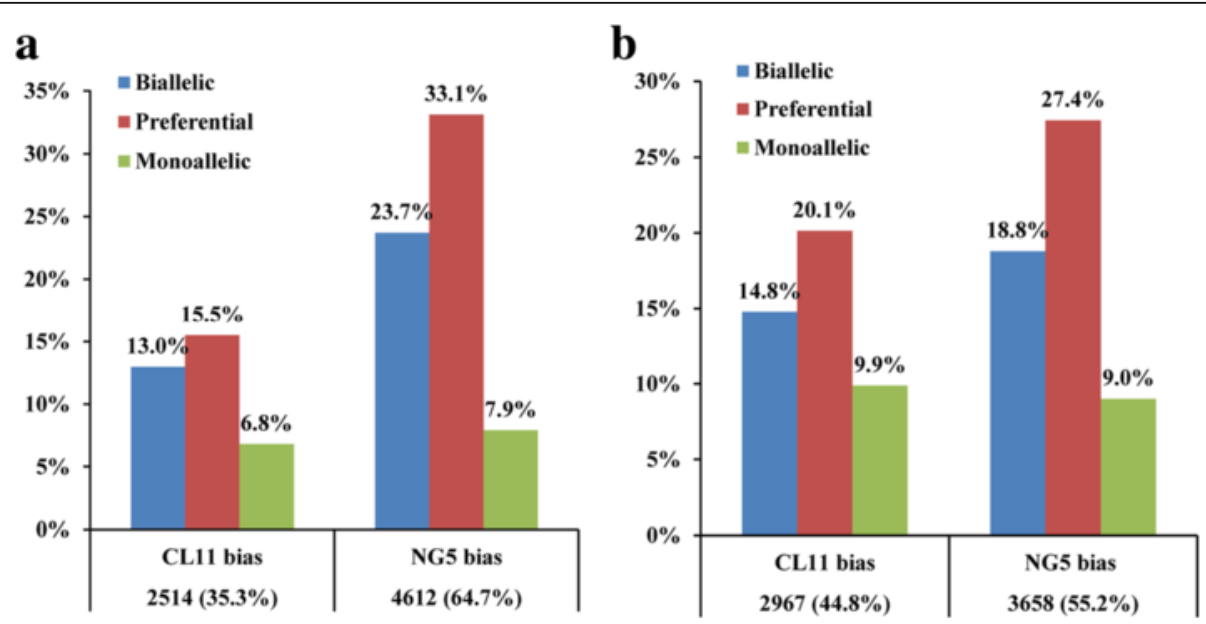

Fig. 4 The allele-specific expression analysis in hybrid in the spikelet and floret differentiation stages. The proportions of genes with monoallelic expression, preferential allelic expression and biallelic expression profiles in hybrid in the spikelet (a) and floret differentiation stage (b) of maize ear 

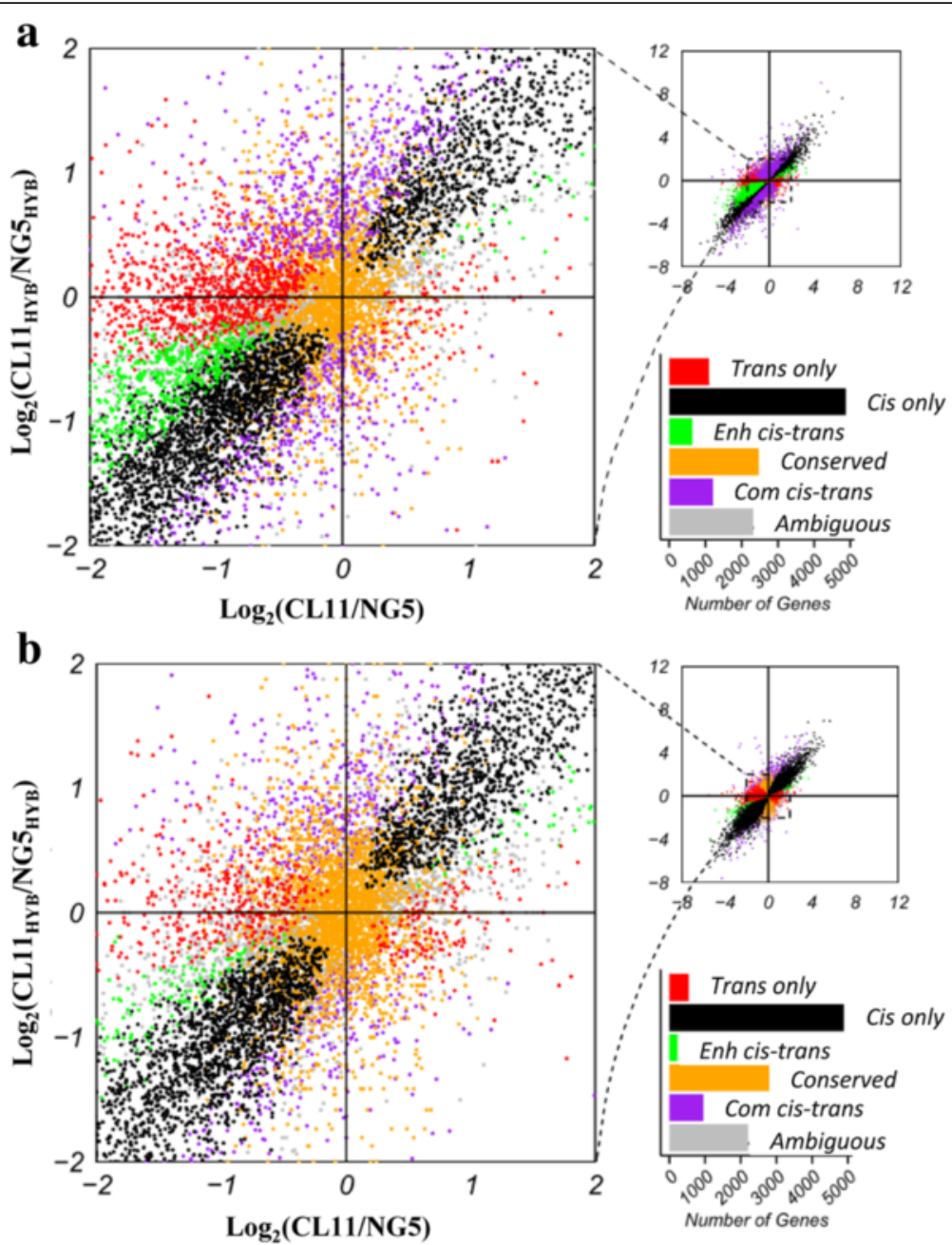

Fig. 5 The Plot summarizes the relative allele-specific expression levels in parental and F1 hybrids. Each point represents a single gene and is color-coded according to the mechanism of regulatory evolution inferred from statistical tests. The bar graph depicts the number of genes in each category in the spikelet (a) and floret differentiation stage (b) of maize ear

divergence in both development stages, which implies that both cis- and trans-regulatory divergence tend to cause a higher expression of the NG5 allele (Additional file 12). This finding is consistent with the high level of NG5 $5_{\mathrm{HYB}}$ allelic bias in the hybrid.

Comparisons between the two developmental stages revealed that, among the 10,388 genes analyzed in both developmental stages, $65.5 \%$ of the cis only genes from the spikelet stage maintained a consistent regulation pattern in the floret stage, but merely $17.8 \%$ of the trans only genes and $26.6 \%$ of the cistrans interaction genes maintained their regulation patterns (Additional file 15).

Relationship between cis- and trans-regulatory divergence and gene expression patterns

Finally, we looked for correlations between gene expression patterns and the mechanism of regulatory divergence.
The absolute magnitude of parental divergence resulting from different regulation categories illustrated that a vast majority of gene expression differences between parents were regulated by cis effects (Fig. $6 a$ and b), and the proportion of total expression divergence regulated by cis effects increased with the magnitude of divergence (Fig. 6c and $\mathrm{d}$ ).

Genes subject to cis-regulatory variation are expected to have additive effects on gene expression in the hybrids [31]. We compared the proportions of genes showing additive and non-additive gene action in the cis only list. More than $65 \%$ of cis genes exhibited additive gene action in the spikelet and floret stages, and 84 to $91 \%$ of cis genes contributed to an additive expression pattern when the $\log _{2}$ ratio of parent expression divergence was greater than 5 . Only a few cis genes displayed a dominant and over/under-dominant expression pattern (Fig. 7a and b). 
$\mathbf{a}$

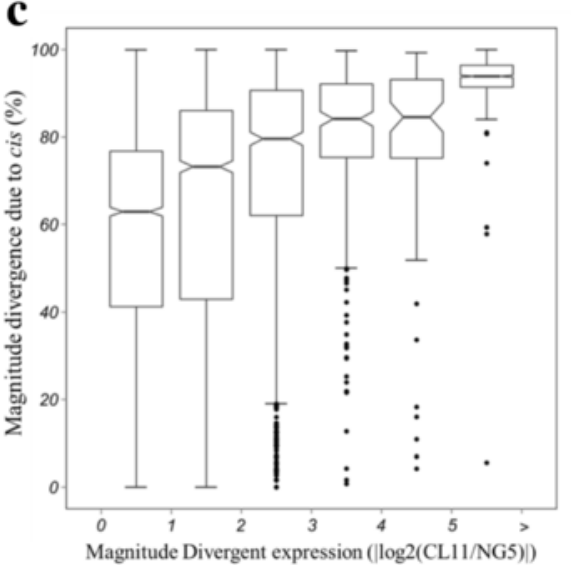

b

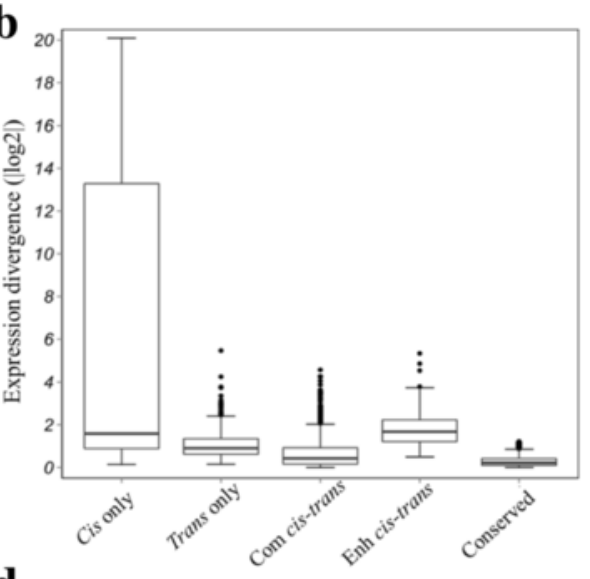

d

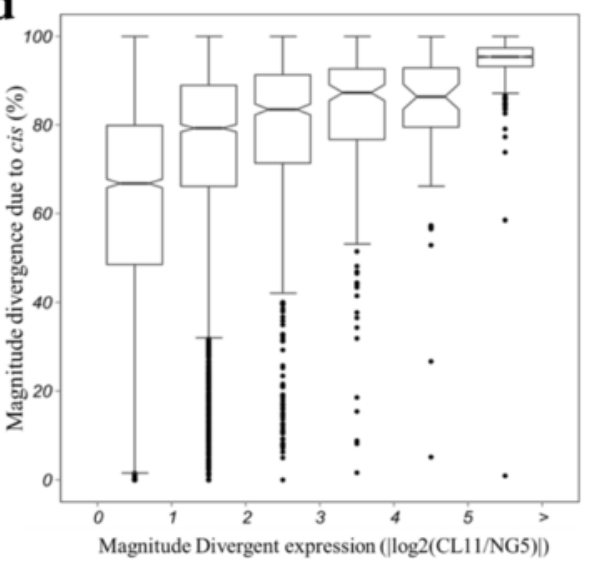

Fig. 6 The relative contributions of cis- and trans-regulation to variation in gene expression. Absolute magnitude (fold-change) of parental divergence resulting from cis only, trans only, compensating (Com) and enhancing (En) cis and trans interaction in the spikelet differentiation stage (a) and floret differentiation stage (b) of maize ear. Box-and-whisker plots showing the percent of cis-effects for genes binned based on the magnitude of expression divergence between parents in the spikelet differentiation stage (c) and floret differentiation stage (d) of maize ear
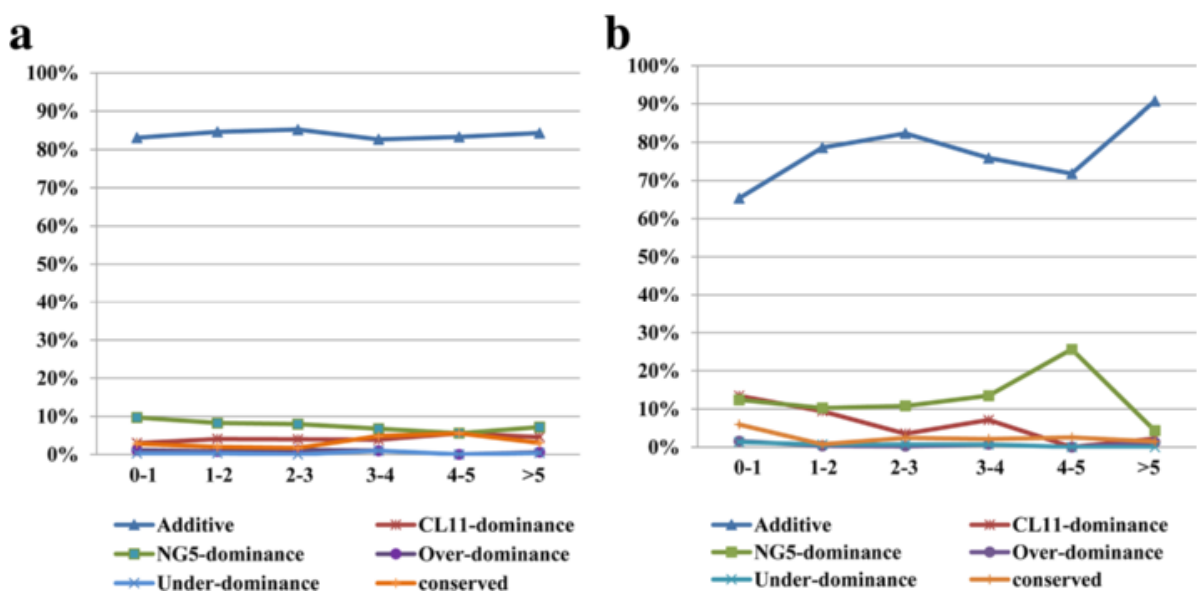

Fig. 7 The percentage of cis-effects for genes showing additive and non-additive inheritance. Distributions of percent cis for genes showing additive, CL11-dominance, NG5-dominance, over-/under-dominance and conserved inheritance in the spikelet differentiation stage (a) and floret differentiation stages $(\mathbf{b})$ of maize ear. Total divergence ( $\log _{2}$ of parent expression ratio) was binned from $0-1,1-2,2-3,3-4,4-5$ and $>5$ 


\section{SNP confirmation and qRT-PCR validation of differentially expressed genes from RNA-seq}

To confirm the accuracy and reproducibility of the RNA-seq results, 17 differentially expressed genes were randomly selected for real-time PCR (qRT-PCR), and the correlation between RNA-seq and qRT-PCR was evaluated using $\log _{2}$-fold change measurements. The qRT-PCR results showed that the expression trends of these genes were significantly similar $\left(\mathrm{r}^{2}=0.83\right)$ to those of the RNA-seq data (Fig. 8 and Additional file 16). To verify the accuracy of SNPs used in ASE analysis, 115 SNPs from 35 genes were amplified from each genotype using gDNA and cDNA as PCR templates, and the PCR products were sequenced with the Sanger method. All the SNPs were confirmed correctly (Additional file 17).

\section{Discussion}

\section{Prevalent differential gene expression among ZD808 and} its parental lines

Maize ear inflorescence development is a complex and dynamic biological process involving different regulatory networks and a large number of genes. Our RNA sequencing results revealed that 21,258 genes $(53.9 \%$ of maize protein coding genes) were transcribed in at least one genotype (Additional file 3). This finding indicates the active gene regulation underlying immature ear development. More genes were expressed in the hybrid compared to CL11 and NG5 in both developmental stages, suggesting that complementation contributes to transcriptome complexity in hybrids and helps explain hybrid phenotypic advantages.

Previous studies have suggested a correlation between heterosis and gene expression variation. Substantial numbers of differentially expressed genes were discovered among the hybrids and their parents in many species $[15,17,31,51]$. Using microarray technology, 4$18 \%$ of expressed genes were identified as being significantly differentially expressed in different genetic backgrounds of maize immature ear, seedling and embryo tissues [15, 31]. In rice, $10.6 \%$ of the total gene set is differentially expressed in the super hybrid LYP9 and its parental cultivars 93-11 and PA64s [51]. RNA-seq technology revealed higher proportions of DEGs among the cultivars Nipponbare, 93-11 and their reciprocal F1 hybrids [17]. A recent report revealed that nearly $70 \%$ of maize expressed genes were differentially expressed between B73 and Mo17, and $42-55 \%$ were differentially expressed between hybrids and their parents [20]. In our study, 54.5 and $43.9 \%$ DEGs were identified between CL11 and NG5 in the spikelet and floret differentiation stages of maize ear (Table 2). The relatively high percentages of DEGs between CL11 and NG5 demonstrated the large genetic distance between the two parental lines, which may be an important reason for the superior performance of ZD808. Analysis of gene expression differences between the hybrid and its parental lines revealed that more DEGs occurred between the hybrid and CL11 (36.0 and 31.8\%) compared with the DEGs between the hybrid and NG5 (18.7 and 11.7\%) in both developmental stages (Table 2). These data suggest that gene expression in the hybrid is more similar to the paternal line NG5. This result is further confirmed by hierarchical clustering analysis (Fig. 2c). Observation of the ear phenotypes of CL11 and NG5 showed that significant $\mathrm{MPH}$ and $\mathrm{BPH}$ were observed in both development stages, and the paternal line NG5 was more vigorous than CL11 (Fig. 1a and b and Table 1). Thus, we can further deduce that NG5 may play an important role in the ear heterosis of ZD808.

In the spikelet differentiation stage, the MPH and $\mathrm{BPH}$ values of the ear length and ear diameter were comparatively higher compared to those in the floret differentiation stage (Table 1). Comparisons between the two developmental stages revealed more DEGs between ZD808 and its two parents in the spikelet differentiation stage, which implies that the DEGs may be positively correlated with the degree of heterosis.
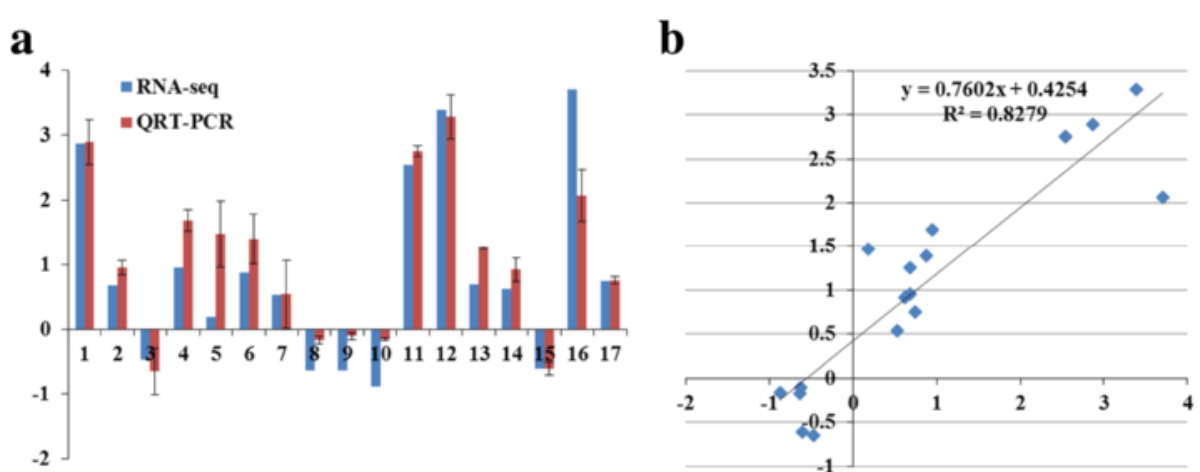

Fig. 8 Correlation between qRTPCR and RNA-seq with selected differentially expressed genes. a qRT-PCR validation of differentially expressed genes in the spikelet and floret differentiation stages from RNA-seq. $\mathbf{b}$ Correlation analysis in log2 fold change measurement between RNA-seq and qRT-PCR. 


\section{Additive gene expression patterns play fundamental roles in maize ear heterosis}

The debate regarding the relationship between heterosis and additive and non-additive gene expression has been discussed in previous studies. Using immature ear tissues of a series of 16 maize hybrids as materials, Guo et al. revealed that the proportion of additively expressed genes is positively associated with hybrid yield and heterosis, whereas non-additively expressed genes are negatively correlated or not correlated with either yield or heterosis [14]. Swanson-Wagner et al. reported that only $\sim 25 \%$ of differentially expressed maize genes exhibited non-additive expression profiles; the vast majority of them were expressed within the range of the two parents [31]. Similar findings were also reported by Stupar et al., who found no obvious correlation between non-additive expression and different heterosis levels [16]. Using RNA-seq, Paschold et al. discovered that only $10 \%$ of analyzed genes were non-additively expressed [20]. However, in other studies, genes with dominant or transgressive expression were more prevalent and considered to be important in conferring novel or superior hybrid performance. Auger et al. revealed that a substantial number of genes are not expressed at the midparent level in maize hybrids [24]. In maize internodes, over $50 \%$ of expressed genes showed an over-dominant gene expression pattern, and only $10.2 \%$ showed additive gene action [21]. A recent study of nascent allohexaploid wheat revealed that a high proportion of protein-coding genes exhibit parental expression level dominance and contribute to growth vigor [19]. In certain other species, transgressive expression seems to be especially common [32, 52, 53].

Consistent with previous studies, our results support the notion that multiple gene expression patterns exist between ZD808 and its parental lines. Among all the gene expression models, 73.8 and $79.8 \%$ of genes were additively expressed in the hybrid (Table 3), which suggests that the complementary interaction of two parental alleles occurred in the hybrid for most genes. This complementary effect might neutralize the effect of deleterious alleles and adjust the gene expression level into an optimal status. Springer et al. posited a similar hypothesis that the mid-parent expression pattern may increase the fitness in hybrid and play a foundation role in heterosis [25]. In addition, 26.2-20.1\% of genes displayed non-additivity expression pattern. Of these, more than $80 \%$ were expressed at parental-like levels, and 55.470.5\% were NG5-dominant genes (Table 3). These results suggest that the NG5 may provide many advantageous alleles that confer specific functions in maize ear development and lead to heterosis.

GO enrichment analysis revealed that additively expressed genes were mainly overrepresented in basic biological process and molecular functions in both development stages. In the biological process category, 'metabolic process' (54.0 and 55.4\%), 'cellular process' (53.2 and 53.0\%) and 'biological regulation' (18.3 and $19.1 \%$ ) were the most highly represented GO categories. Under the 'metabolic process' and 'cellular process' GO terms, 'primary metabolic process' (including 'carbohydrate metabolic process' and 'protein metabolic process'), 'cellular metabolic process' (including 'cellular macromolecule metabolic process' and 'cellular biosynthetic process'), 'macromolecule metabolic process' (including “protein metabolic process', 'gene expression' and 'macromolecule biosynthetic process') and 'biosynthetic process' (including 'cellular biosynthetic process' and 'cell macromolecule biosynthetic process') were found enriched in both stages (Fig. 3b Additional file 7). These results indicated that additively expressed genes were functioned in carbohydrate, protein and cellular macromolecule biosynthesis and metabolism in both stages, which are essential processes that produce both structural components and energy sources for maize ear development and heterosis formation.

While the non-additive expressed genes differently enriched between spikelet and floret differentiation stages. Among the NG5-dominant genes, the BP terms 'nitrogen compound metabolic process' (22.4\%) and the MF terms 'nucleotide binding' (20.0\%) were significantly enriched in the spikelet differentiation stage, but only "catalytic activity" (50.7\%) was enriched in the floret differentiation stage (Fig. 3c and Additional file 7). Further inspection of the NG5-dominant genes revealed MADSbox, bHLH DNA-binding superfamily protein, Auxin response factors and Ethylene-responsive transcription factors in the 'cellular process' category (Additional file 8), these genes play important roles in coordinating the growth and differentiation of cells into new organs and regulating auxin or ethylene signaling to promote the transition from SPMs to SMs. Nitrogen (N) is crucially required for maize ear development, and $\mathrm{N}$ deficiency may reduce the kernel number and decrease the grain yield [54]. Many genes were discovered involving in glutamine synthesis, glutamine metabolism and aspartate metabolism in the 'nitrogen compound metabolic process' term. These genes may be essentially important for nitrogen assimilation and affect maize ear development. In the floret differentiation stage, genes involved in oxidation-reduction reactions were found in the 'catalytic activity' term; these genes may mainly participate in stress responses and signal transduction (Additional file 8). These results indicated that additive expressed genes were fundamentally required for ear heterosis formation in both development stages, while NG5-dominant genes may contribute to the stage-specific vigor phenotype. 


\section{Allele-specific expression contributes to differential gene expression}

Allelic variation is widespread in the maize genome, and combinations of parental allelic variants in hybrids may result in novel patterns of gene expression and contribute to superior phenotypes. Allelic expression bias was consistently observed for 50 and $60 \%$ of genes assayed in maize hybrid seedlings and meristems $[27,55]$. In the rice hybrid Xieyou9308, 17\% of transcripts showed significant allelic bias at the tillering and heading stages [56]. A similar study of a reciprocal F1 hybrid between rice Nipponbare and $93-11$ revealed that $22.7 \%$ of genes exhibited significant allelic expression differences [57]. In Arabidopsis, about 40\% of genes showed allelic expression differences in the hybrid [58]. In our study, the global ASE profile of the ear of ZD808 indicates that 56.4 and $52.4 \%$ of analyzed genes exhibited significant allelic expression bias in the spikelet and floret differentiation stages, respectively (Fig. 4a and b). The higher rate of ASE in our results suggests that a large number of allelic variations may exist between CL11 and NG5.

Our results also show that the majority of ASE genes (64.7 and 55.2\%) displayed NG5 $5_{\mathrm{HYB}}$ allelic expression bias in both developmental stages (Fig. 4a and b). The results suggest that the NG5 genome contributes greatly to the activity of the transcriptomes in the hybrid and explains the high level of NG5-dominant expression. A comparison of the ASE pattern between the two development stages revealed that $30.1 \%$ of ASE genes in the spikelet differentiation stages changed their allelic bias pattern, which indicates that the parental alleles in hybrids may exhibit cisregulatory variation that result in differential responses to development cues (Additional file 11).

\section{Cis- and trans-regulatory differences underlying gene expression novelty}

Quantitative changes in allele-specific expression may be the result of cis- and/or trans-regulatory variations $[28,59]$. In our research, in the spikelet and floret developmental stages, respectively, 38.5 and $40.8 \%$ of analyzed genes were affected by cis only regulatory divergence, 8.8 and $4.6 \%$ of expressed genes were affected by trans only effects, and 14.8 and $10.0 \%$ of genes showed evidence of both (Fig. $5 \mathrm{a}$ and $\mathrm{b}$ and Additional file 12). The prevalence of cis-regulatory variation reflects the frequency of allelic variation in the maize genome and suggests the maintenance of inbred allelic expression levels in the hybrid. We also found that both cis- and trans-regulation tend to drive higher expression of the NG5 alleles, which explains the high level of NG5 $5_{\text {HYB }}$ bias in the hybrid and the NG5-dominant expression pattern. The cis and trans effect genes were further classified into enhancing and compensating interactions. Previous studies have revealed that stabilizing selection is characterized by compensating cis and trans effects and diversifying selection corresponds to enhancing cis and trans effects [34,60]. In our study, 60.7 and $80.3 \%$ of cisand trans-regulation genes, respectively, exhibited compensating interaction in both development stages (Fig. $5 \mathrm{a}$ and $\mathrm{b}$ and Additional file 12), which suggests a prevalent role for stabilizing selection in maintaining gene expression levels.

Further analysis of the absolute magnitude of parental divergence resulting from different regulation categories illustrated that the vast majority of gene expression divergences between parents were regulated by cis effects (Fig. 6a and b), and the proportion of total expression divergence regulated by cis effects increased withthe magnitude of divergence (Fig. $6 \mathrm{c}$ and d). Taken together, these data indicate that cis-regulatory effects play a larger role than trans effects. Some studies have found that cis-regulatory variation is positively correlated with additive expression patterns in the F1 hybrid [30, 31]. Our study found that 65 to $91 \%$ of cis-regulated genes exhibit additive gene activity in the spikelet and floret differentiation stages (Fig. $7 \mathrm{a}$ and $\mathrm{b}$ ). These data provide evidence that prevalent cis-regulatory variations contribute to allelic expression bias and result in an additive expression pattern in the maize hybrid ZD808.

Between the two stages, only $65.5 \%$ of cis only genes, $17.8 \%$ of trans only genes and $26.6 \%$ of cis-trans interaction genes maintained a consistent regulatory pattern (Additional file 15), which suggests that developmental stage-specific cis-/trans-regulation may explain the differential gene expression patterns between the two stages and lead to different manifestations of ear heterosis. Previous studies have also reported that cis-/transregulated variation shows differential responses to environmental [30] or developmental signals [61, 62].

\section{Conclusion}

Using RNA sequencing technology, we systematically investigated the global transcriptomes of maize ear from the hybrid ZD808 and its parents in the spikelet and floret differentiation stages. Our results demonstrated that additive gene expression patterns were prevalent in the hybrid in both development stages, which suggested that the complementary interaction of two parental alleles occurred in the hybrid for most genes. This complementary effect may adjust the gene expression level into an optimal status and play a foundation role in maize ear heterosis. Among the non-additively expressed genes in the hybrid, the majority of which were expressed at NG5 dominant level, indicating that paternal line NG5 may provide beneficial alleles contributing to hybrid vigor. Analysis of allele-specific expression patterns in the hybrid suggested that variation in gene expression levels was largely attributable to cis-regulatory variation in maize. The cis-regulatory variations tend to preserve the allelic expression levels in hybrid and cause 
additive expression. Comparison between the two stages revealed that allele-specific expression and cis-/transregulatory variations responded differently to developmental cues, which may lead to different degree of heterosis during maize ear development.

Therefore, our work provides a comprehensive insight into transcriptional variation and its correlation with heterosis during maize ear development. The findings improve our understanding of the molecular basis of heterosis in maize and present novel opportunity to improve our maize varieties in the future.

\section{Methods}

\section{Sample preparation and transcriptome sequencing}

ZD808 (HYB) and its parental lines CL11 and NG5 were grown in experimental fields in the spring of 2013 under regular farming conditions in Nanchong, Sichuan. Ears were manually collected at the spikelet and floret differentiation stages according to the leaf age index combined with scanning electron microscopy. Five immature ears were pooled for the two biological replicates per genotype and were ground in liquid nitrogen.

Total RNA was extracted from each sample by using TRIzol reagent (Invitrogen, CA, USA). RNA degradation and contamination were monitored on $1 \%$ agarose gels. RNA quality was assessed using the Bioanalyzer 2100 system (Agilent Technologies, CA, USA) with a minimum RNA integrity number (RIN) of 7.0.

A 3- $\mu$ g RNA sample was used as input material for library preparation. Sequencing libraries were generated using the NEBNext ${ }^{\circ}$ Ultra $^{\text {Tu }}$ RNA Library Prep Kit for Illumina ${ }^{\circ}$ (NEB, USA), and index codes were assigned to each sample. Library quality was assessed on the Agilent Bioanalyzer 2100 system. The clustering of the index-coded samples was performed on a cBot Cluster Generation System using the TruSeq PE Cluster Kit v3-cBot-HS (Illumina). After cluster generation, the prepared libraries were sequenced on an Illumina HiSeq 2000 platform and 100 bp paired-end reads were generated.

\section{Sequenced read processing, alignment and gene expression level quantification}

The raw reads were filtered before data analysis by removing reads consisting of adaptors, reads with more than $10 \% \mathrm{~N}$, and low-quality sequences (more than $50 \%$ of the reads having a phred base $s Q \leq 5$ ). The paired-end clean reads were aligned to the B73 reference genome (RefGen_v3) using the default parameters of TopHat v2.0.9. Reference genome and gene model annotation files were downloaded from genome website (http:// ensembl.gramene.org/Zea_mays/Info/Index) directly.

HTSeq v0.5.4p3 was used to count the read numbers mapped to each gene. The RPKM of each gene was calculated based on the length of the gene, and read counts were mapped to the gene.

\section{Identification and classification of differential gene expression patterns}

Differential expression analysis was performed using the $\mathrm{R}$ package DESeq. For all comparisons, the resulting $p$ values were adjusted using Benjamini and Hochberg's approach for controlling the false-discovery rate. Genes that exhibited an adjusted $p$-value $<0.05$ (q-value) were determined to be significantly differentially expressed. To gain overall insight into gene expression inheritance patterns in the F1 hybrids and parental lines, the gene expression levels in the hybrids were compared to the mid-parent expression level. Genes with q-value $<0.05$ were regarded as non-additive. Genes with q-value $>0.05$ were regarded as additive. The non-additive genes were further classified into more specific categories. Genes with an F1 genotype mean that was not significantly different from one parent but was significantly higher (or lower) than the other parent were considered to exhibit high-parent (low-parent) dominance. Genes with an F1 genotype mean that was significantly higher (or lower) than both of the inbred line parents were said to exhibit over-dominance (under-dominance).

\section{SNP identification and allele-specific expression analysis}

Picard-tools v1.96 and SAMtools v0.1.18 were used to sort and mark duplicated reads and reorder the bam alignment results of each sample. GATK2 software was used to perform SNP calling. Unreliable SNPs between CL11 and NG5 were filtered out according to the following criteria: 1) all reads uniquely match both CL11 and NG5 genomes, and the read quality value is no lower than $20 ; 2$ ) all reads from one parent produce a consensus base at the SNP position but different from another parent; 3) the SNP is represented by at least 10 reads. Allelic bias in hybrids was identified by determining for each SNP whether there was significant deviation from the binomial distribution of parental alleles (i.e., the allele ratio in the hybrids deviated from 1.0).

\section{Cis- and trans-regulatory effects}

To estimate the relative contributions of cis- and transregulatory factors, we performed statistical tests to compare the ratios of expression of the two parental alleles in the hybrid with the relative expression level of the consistent allele in the parental lines, as described in a previous study [32]. The overall gene expression divergence was quantified as $\log _{2}$ (CL11/NG5) (A) using the binomial exact test, with the $p$-value corrected by the FDR method. The significant cis-effects, referred to as allelic imbalance in the hybrid, were also determined with the binomial exact test. The extent of the cis-effects 
was quantified as $\log _{2}\left(\mathrm{CL} 11_{\mathrm{HYB}} / \mathrm{NG} 5_{\mathrm{HYB}}\right)$ (B). Fisher's exact test followed by FDR analysis was used to divide trans-effects by subtracting cis-effects from the expression divergence between CL11 and NG5 (A-B). Based on the significance test, the genes could be categorized into five classes: 1) Cis Only: significant expression differences in $\mathrm{A}$ and $\mathrm{B}$; no significant $\mathrm{A}-\mathrm{B}$; 2) trans Only: significant expression in A but not $\mathrm{B}$; significant A-B; 3) cis and trans effects (cis-trans): Significant differential expression in A and B; significant A-B; 4) Conserved: No significant differential expression in $A$ or $B$; no significant A-B; 5) Ambiguous: All other patterns of significance tests, with no clear biological interpretation.

The cis-trans category can be further divided into Compensating and Enhancing cis and trans interactions. The Enhancing cis-trans interaction was implied if the $\log _{2}$-transformed allele-specific ratios of these genes in the parental and hybrid data sets had the same direction. If they were in the opposite direction, the interaction was Compensating.

\section{Additional files}

Additional file 1: Table S1. RNA-seq data statistics and genome mapping rates. (XLSX $16 \mathrm{~kb}$ )

Additional file 2: Figure S1. Pearson's correlation coefficient between two biological replicates of each genotype calculated using $\log _{10}(\mathrm{RPKM}+1)$ Figure S2. Analysis of genes differentially expressed between the spikelet and floret differentiation stages in three genotypes. Figure S3. Directed Acyclic Graph (DAG) visualization for Gene Ontology enrichment of 249 differentially expressed genes. (DOCX 1694 kb)

Additional file 3: Table S2. Distribution of gene expression levels in each genotype. (DOCX $14 \mathrm{~kb}$ )

Additional file 4: Table S3. DEGs involved in regulation of ear development from the 'developmental process' and 'biological regulation' terms. (XLSX $16 \mathrm{~kb}$ )

Additional file 5: Dataset S1. The differential gene expression patterns in the spikelet differentiation stage. (XLSX $3916 \mathrm{~kb}$ )

Additional file 6: Dataset S2. The differential gene expression patterns in the floret differentiation stage. (XLSX $3226 \mathrm{~kb}$ )

Additional file 7: Table S4. Cross-comparison of enriched GO terms of additive and non-additive expressed genes in the spikelet and floret differentiation stages. (XLSX $30 \mathrm{~kb}$ )

Additional file 8: Table S5. The selected NG5-dominant expressed genes that were assigned to different $\mathrm{GO}$ terms in the spikelet and floret differentiation stages. (DOCX $20 \mathrm{~kb}$ )

Additional file 9: Dataset S3. ASE patterns in hybrid in the spikelet differentiation stage. (XLSX $1350 \mathrm{~kb}$ )

Additional file 10: Dataset S4. ASE patterns in hybrid in the floret differentiation stage. (XLSX $1280 \mathrm{~kb}$ )

Additional file 11: Table S6. Comparison of the ASE patterns in hybrid between the two development stages. (DOCX $16 \mathrm{~kb}$ )

Additional file 12: Table S7. Gene numbers of different cis- and transregulatory categories. (DOCX $15 \mathrm{~kb}$ )

Additional file 13: Dataset S5. Cis- and trans-regulatory variations in the spikelet differentiation stage. (XLSX $1351 \mathrm{~kb}$ )

Additional file 14: Dataset S6. Cis- and trans-regulatory variations in the floret differentiation stage. (XLSX $1280 \mathrm{~kb}$ )
Additional file 15:Table S8. Comparison of the cis- and transregulatory variations between the spikelet and floret differentiation stages. (DOCX $14 \mathrm{~kb}$ )

Additional file 16: Table S9. qRT-PCR validation of differentially expressed genes in the spikelet and floret differentiation stages from RNA-seq. (DOCX $20 \mathrm{~kb}$ )

Additional file 17: Table S10. Verified SNPs locus in ASE analysis. (DOCX $37 \mathrm{~kb})$

\section{Abbreviations}

ASE: Allele-specific expression; BPH: Best-parent heterosis; DEGs: Differentially expressed genes; FDR: False discovery rate; FM: Floret meristem; Fstage: Floret differentiation stage; GO: Gene ontology; IM: Inflorescence meristem; MPH: Mid-parent heterosis; RNA-Seq: RNA sequencing technology; RPKM: Reads per kilobase per million reads; SM: Spikelet meristem; SNP: Single-nucleotide polymorphism; SPM: Spikelet pair primordia; Sstage: Spikelet differentiation stage

\section{Acknowledgement}

We thank professors Long Mao, Aili Li, Jun Liu and wenxue Li for constructive suggestions and the two anonymous reviewers for helpful comments. We also thank the Novogene Bioinformatics Institute for conducting RNA-Seq using Illumina sequencing platform.

\section{Funding}

This work was supported by funds from the National Basic Research Program of China (973 Program; 2014CB138200) and The National High Technology Research and Development Program of China (863 Program; 2011AA10A103) and the Agricultural Science and Technology Innovation Program at CAAS.

\section{Availability of data and materials}

The datasets supporting the results of this article are included within the article and its additional files. Illumina sequences were deposited to the NCBI Sequence Read Archive under the accessions SRP066518 (http:// www.ncbi.nlm.nih.gov/sra/ SRP066518).

\section{Authors' contributions}

HXJ, WHW and HCL conceived and designed the experiments. WYJ conducted the field experiments and collected the tissue samples. HXJ WHW and DXZ performed the experiment and analysed the data. HXJ interpreted the data and drafted the manuscript. WHW, LZF and HCL supervised the project and revised the manuscript. LK, LQJ and WH provided helpful discussions on the manuscript. All authors read and approved the final manuscript.

\section{Competing interests}

The authors declare that they have no competing interests.

\section{Consent for publication}

Not applicable.

Ethics approval and consent to participate Not applicable.

Received: 25 January 2016 Accepted: 15 November 2016 Published online: 22 November 2016

References

1. Chen ZJ. Genomic and epigenetic insights into the molecular bases of heterosis. Nat Rev Genet. 2013;14:471-82.

2. Schnable PS, Springer NM. Progress toward understanding heterosis in crop plants. Annu Rev Plant Biol. 2013;64:71-88.

3. Lippman ZB, Zamir D. Heterosis: revisiting the magic. Trends Genet. 2007;23:60-6.

4. Duvick DN. Biotechnology in the 1930s: the development of hybrid maize Nat Rev Genet. 2001;2:69-74.

5. Bruce A. The Mendelian theory of heredity and the augmentation of vigor. Science. 1910;32:627-8.

6. Jones DF. Dominance of linked factors as a means of accounting for heterosis. Genetics. 1917;2:466. 
7. Crow JF. Alternative hypotheses of hybrid vigor. Genetics. 1948;33:477.

8. Powers L. An expansion of Jones's theory for the explanation of heterosis. Am Nat. 1944;78:275-80.

9. Williams W. Heterosis and the genetics of complex characters. Heredity. 1960;15:327-8.

10. Hochholdinger F, Hoecker N. Towards the molecular basis of heterosis. Trends Plant Sci. 2007;12:427-32.

11. Birchler JA, Auger DL, Riddle NC. In search of the molecular basis of heterosis. Plant Cell. 2003;15:2236-9.

12. Miller M, Zhang C, Chen ZJ. Ploidy and hybridity effects on growth vigor and gene expression in Arabidopsis thaliana hybrids and their parents. G3 GENES GENOM GENET. 2012;2:505-13.

13. Wang J, Tian L, Lee H-S, Wei NE, Jiang H, Watson B, et al. Genomewide nonadditive gene regulation in Arabidopsis allotetraploids. Genetics. 2006; 172:507-17.

14. Guo M, Rupe MA, Yang X, Crasta O, Zinselmeier C, Smith OS, et al. Genomewide transcript analysis of maize hybrids: allelic additive gene expression and yield heterosis. Theor Appl Genet. 2006;113:831-45.

15. Swanson-Wagner RA, Jia Y, DeCook R, Borsuk LA, Nettleton D, Schnable PS. All possible modes of gene action are observed in a global comparison of gene expression in a maize F1 hybrid and its inbred parents. Proc Natl Acad Sci U S A. 2006;103:6805-10.

16. Stupar RM, Gardiner JM, Oldre AG, Haun WJ, Chandler VL, Springer NM. Gene expression analyses in maize inbreds and hybrids with varying levels of heterosis. BMC Plant Bio. 2008:8:33.

17. He G, Zhu X, Elling AA, Chen L, Wang X, Guo L, et al. Global epigenetic and transcriptional trends among two rice subspecies and their reciprocal hybrids. Plant Cell. 2010;22:17-33.

18. Song G-S, Zhai H-L, Peng Y-G, Zhang L, Wei G, Chen X-Y, et al. Comparative transcriptional profiling and preliminary study on heterosis mechanism of super-hybrid rice. Mol Plant. 2010;3:1012-25.

19. Li A, Liu D, Wu J, Zhao X, Hao M, Geng S, et al. mRNA and small RNA transcriptomes reveal insights into dynamic homoeolog regulation of allopolyploid heterosis in nascent hexaploid wheat. Plant Cell. 2014;26:1878-900.

20. Paschold A, Jia Y, Marcon C, Lund S, Larson NB, Yeh C-T, et al. Complementation contributes to transcriptome complexity in maize (Zea mays L.) hybrids relative to their inbred parents. Genome Res. 2012;22:2445-54.

21. Użarowska A, Keller B, Piepho H-P, Schwarz G, Ingvardsen C, Wenzel G, et al Comparative expression profiling in meristems of inbred-hybrid triplets of maize based on morphological investigations of heterosis for plant height. Plant Mol Biol. 2007;63:21-34

22. Song $\mathrm{S}, \mathrm{Qu} \mathrm{H}, \mathrm{Chen} \mathrm{C}, \mathrm{Hu} \mathrm{S}, \mathrm{Yu}$ J. Differential gene expression in an elite hybrid rice cultivar (Oryza sativa, L) and its parental lines based on SAGE data. BMC Plant Biol. 2007;7:49.

23. Song $\mathrm{R}$, Messing J. Gene expression of a gene family in maize based on noncollinear haplotypes. Proc Natl Acad Sci U S A. 2003;100:9055-60.

24. Auger DL, Gray AD, Ream TS, Kato A, Coe EH, Birchler JA. Nonadditive gene expression in diploid and triploid hybrids of maize. Genetics. 2005;169:389-97.

25. Springer NM, Stupar RM. Allelic variation and heterosis in maize: how do two halves make more than a whole? Genome Res. 2007;17:264-75.

26. Birchler JA, Yao H, Chudalayandi S, Vaiman D, Veitia RA. Heterosis. Plant Cell. 2010;22:2105-12.

27. Springer NM, Stupar RM. Allele-specific expression patterns reveal biases and embryo-specific parent-of-origin effects in hybrid maize. Plant Cell. 2007:19:2391-402

28. Wittkopp PJ, Haerum BK, Clark AG. Evolutionary changes in cis and trans gene regulation. Nature. 2004;430:85-8.

29. Wittkopp PJ, Haerum BK, Clark AG. Independent effects of cis-and transregulatory variation on gene expression in Drosophila melanogaster. Genetics. 2008;178:1831-5.

30. Guo M, Rupe MA, Zinselmeier C, Habben J, Bowen BA, Smith OS. Allelic variation of gene expression in maize hybrids. Plant Cell. 2004;16:1707-16.

31. Stupar RM, Springer NM. Cis-transcriptional variation in maize inbred lines B73 and Mo17 leads to additive expression patterns in the F1 hybrid. Genetics. 2006;173:2199-210.

32. McManus CJ, Coolon JD, Duff MO, Eipper-Mains J, Graveley BR, Wittkopp PJ. Regulatory divergence in Drosophila revealed by mRNA-seq. Genome Res. 2010;20:816-25.

33. Emerson J, Hsieh L-C, Sung H-M, Wang T-Y, Huang C-J, Lu HH-S, et al, Natural selection on cis and trans regulation in yeasts. Genome Res. 2010;20:826-36
34. Shi X, Ng DW, Zhang C, Comai L, Ye W, Chen ZJ. Cis- and trans-regulatory divergence between progenitor species determines gene-expression novelty in Arabidopsis allopolyploids. Nature Commun. 2012;3:950.

35. Combes M-C, Hueber $Y$, Dereeper A, Rialle S, Herrera J-C, Lashermes P. Regulatory divergence between parental alleles determines gene expression patterns in hybrids. Genome Biol Evol. 2015;7:1110-21.

36. Zhu Y, Fu J, Zhang J, Liu T, Jia Z, Wang J, et al. Genome-wide analysis of gene expression profiles during ear development of maize. Plant Mol Biol. 2009;70:63-77.

37. Bommert P, Satoh-Nagasawa N, Jackson D, Hirano H-Y. Genetics and evolution of inflorescence and flower development in grasses. Plant Cell Physiol. 2005;46:69-78.

38. Pautler M, Tanaka W, Hirano H-Y, Jackson D. Grass meristems I: shoot apical meristem maintenance, axillary meristem determinacy and the floral transition. Plant Cell Physiol. 2013:54:302-12.

39. Vollbrecht E, Springer PS, Goh L, Buckler IV ES, Martienssen R. Architecture of floral branch systems in maize and related grasses. Nature. 2005;436:1119-26.

40. Bortiri E, Chuck G, Vollbrecht E, Rocheford T, Martienssen R, Hake S. ramosa2 encodes a LATERAL ORGAN BOUNDARY domain protein that determines the fate of stem cells in branch meristems of maize. Plant Cell. 2006;18:574-85.

41. Satoh-Nagasawa N, Nagasawa N, Malcomber S, Sakai H, Jackson D. A trehalose metabolic enzyme controls inflorescence architecture in maize. Nature. 2006;441:227-30.

42. George C, Michael M, Elizabeth K, Sarah H, Schmidt RJ. The control of spikelet meristem identity by the branched silkless 1 gene in maize. Science. 2002:298:1238-41.

43. Mcsteen P, Malcomber S, Skirpan A, Lunde C, Wu X, Kellogg E, et al. barren inflorescence2 Encodes a co-ortholog of the PINOID serine/threonine kinase and is required for organogenesis during inflorescence and vegetative development in maize. Plant Physiol. 2007;144:1000-11.

44. Hubbard L, Mcsteen P, Doebley J, Hake S. Expression patterns and mutant phenotype of teosinte branched 1 correlate with growth suppression in maize and teosinte. Genetics. 2002;162:1927-35.

45. Bomblies K, Wang RLAmbrose BA, Schmidt RJ, Meeley RB, Doebley J. Duplicate FLORICAULA/LEAFY homologs zfl1 and zfl2 control inflorescence architecture and flower patterning in maize. Development. 2003;130:2385-95

46. Muszynski MG, Dam T, Li B, Shirbroun DM, Hou Z, Bruggemann E, et al. delayed flowering1 Encodes a basic leucine zipper protein that mediates floral inductive signals at the shoot apex in maize. Plant Physiol. 2006;142: 1523-36.

47. Ng M, Yanofsky MF. Function and evolution of the plant MADS-box gene family. Nature Rev Genet. 2001;2:186-95.

48. Thompson BE, Linnea B, Clint W, Hall DH, Hajime S, Robert S, et al. Beardedear encodes a MADS box transcription factor critical for maize floral development. Plant Cell. 2009;21:2578-90

49. Tanaka W, Pautler M, Jackson D, Hirano H-Y. Grass meristems II: inflorescence architecture, flower development and meristem fate. Plant Cell Physiol. 2013:54:313-24.

50. Zhuang Y, Adams KL. Extensive allelic variation in gene expression in Populus F1 hybrids. Genetics. 2007:177:1987-96.

51. Wei G, Tao Y, Liu G, Chen C, Luo R, Xia H, et al. A transcriptomic analysis of superhybrid rice LYP9 and its parents. Proc Natl Acad Sci U S A. 2009;106:7695-701.

52. Grover C, Gallagher J, Szadkowski E, Yoo M, Flagel L, Wendel J. Homoeolog expression bias and expression level dominance in allopolyploids. New Phytol. 2012;196:966-71.

53. Yoo M, Szadkowski E, Wendel J. Homoeolog expression bias and expression level dominance in allopolyploid cotton. Heredity. 2013;110:171-80.

54. Hirel B, Bertin P, Quilleré I, Bourdoncle W, Attagnant C, Dellay C, et al. Towards a better understanding of the genetic and physiological basis for nitrogen use efficiency in maize. Plant Physiol. 2001;125:1258-70.

55. Guo M, Yang S, Rupe M, Hu B, Bickel DR, Arthur L, et al. Genome-wide allele-specific expression analysis using massively parallel signature sequencing (MPSS ${ }^{\mathrm{TM}}$ ) reveals cis- and trans-effects on gene expression in maize hybrid meristem tissue. Plant Mol Bio, 2008:66:551-63.

56. Zhai R, Feng Y, Zhan X, Shen X, Wu W, Yu P, et al. Identification of transcriptome SNPs for assessing allele-specific gene expression in a superhybrid rice Xieyou9308. PLoS One. 2013;8:e60668. 
57. Song G, Guo Z, Liu Z, Cheng Q, Qu X, Chen R, et al. Global RNA sequencing reveals that genotype-dependent allele-specific expression contributes to differential expression in rice F1 hybrids. BMC Plant Biol. 2013;13:221.

58. Cubillos FA, Stegle O, Grondin C, Canut M, Tisné S, Gy I, et al. Extensive cisregulatory variation robust to environmental perturbation in Arabidopsis. Plant Cell. 2014;26:4298-310.

59. Wittkopp PJ, Kalay G. Cis-regulatory elements: molecular mechanisms and evolutionary processes underlying divergence. Nature Rev Genet. 2012;13:59-69.

60. Tirosh I, Reikhav S, Levy AA, Barkai N. A yeast hybrid provides insight into the evolution of gene expression regulation. Science. 2009;324:659-62.

61. Adams KL, Wendel JF. Allele-specific, bidirectional silencing of an alcoho dehydrogenase gene in different organs of interspecific diploid cotton hybrids. Genetics. 2005;171:2139-42.

62. Salvi S, Sponza G, Morgante M, Tomes D, Niu X, Fengler KA, et al. Conserved noncoding genomic sequences associated with a flowering-time quantitative trait locus in maize. Proc Natl Acad Sci U S A. 2007;104:11376-81.

Submit your next manuscript to BioMed Central and we will help you at every step:

- We accept pre-submission inquiries

- Our selector tool helps you to find the most relevant journal

- We provide round the clock customer support

- Convenient online submission

- Thorough peer review

- Inclusion in PubMed and all major indexing services

- Maximum visibility for your research

Submit your manuscript at www.biomedcentral.com/submit
Biomed Central 